\title{
A survey on ICT tools for communication development in children with ASD
}

\author{
Margarida Lucas da Silva ${ }^{1}$, Daniel Gonçalves ${ }^{2}$ \\ ${ }^{1}$ Instituto Superior Técnico, Av. Rovisco Pais, 49, 1050-001 Lisboa, Portugal \\ ${ }^{2}$ INESC-ID, R. Alves Redol, 9, 1000-029 Lisboa, Portugal \\ margarida.lucas.silva@ist.utl.pt,daniel.goncalves@vimmi.inesc.pt
}

\begin{abstract}
Keywords: autism spectrum disorders, information and communication technologies, communication development
Abstract: Several studies using Information and Communication Technologies (ICT) have been carried out over the years, trying to solve problems related with multiple dimensions of the limitations faced by children with Autism Spectrum Disorders (ASD). It is not yet possible to conclude that the use of ICT is more beneficial than the use of alternative or traditional educational approaches for children with ASD. In this paper we are going to look at several studies related with communication development in children with ASD, with the purpose of understanding the approaches available and some of their possible results.
\end{abstract}

\section{INTRODUCTION}

The constant evolution in Information and Communication Technologies (ICT) brought a wide range of new possibilities to help people with learning disabilities access new opportunities for learning, entertainment and personal development. Children with Autism Spectrum Disorders (ASD) are included in this group, and although many approaches have been tested but, few have been adopted or successfully integrated in the daily life of the children, their tutors or caregivers (Putnam and Chong, 2008). Due to the difficulties faced by children with ASD in terms of communication skills, a few studies have been developed throughout the years in order to find solutions to minimize this issue. Several approaches have been tested since there is no formula nor enough empirical evidence on how to develop for such a context. Nowadays, the most commonly used tool with children with ASD is the traditional Picture Exchange Communication System (PECS). PECS is an augmentative communication system, developed to help subjects in quickly acquiring a functional means of communication (Bondy and Frost, 2001). It is versatile and inexpensive, since tutors can create and print the pictures that the children needs. It is used with children who need to communicate, but have some sort of impairment that prevents them from using the normal communication channels, and it is sucessfull in delivering clear messages. This tool is mostly used by people with speech limitations, with the need to communicate and understand actions in an effective way. Although a simple approach, this is the general base for most systems and tools aimed at supporting children in communication.

In this chapter we are going to review some of the most relevant studies in this area, focused on any kind of approach for communication development, and also look at commercially available tools, some of which are not specifically designed for patients with ASD, but are still used in some cases.

\section{ICT STUDIES}

We are going to describe 18 studies, all focused on communication, but with some differences on the targeted communication outcomes. Hailpern et al. (2009) (Hailpern et al., 2009) and Simpson et al. (2004) (Simpson et al., 2004) focus their studies in developing the number of vocalizations, proposing methodologies that force the use of speech. Bosseler and Massaro (2003) (Bosseler and Massaro, 2003), Coleman-Martin et al. (2005) (Coleman-Martin et al., 2005), Hetzroni and Shalem (2005) (Hetzroni and Shalem, 2005), Massaro and Bosseler (2006) (Massaro and Bosseler, 2006) and Moore and Calvert (2000) (Moore and Calvert, 2000) focused on developing the children vocabulary, proposing a set of methodologies that tried to teach word to picture matching. Woodcock and Woolner (2007) (Wood- 
cock and Woolner, 2007), Millen et al. (2011) (Millen et al., 2011), Ohene-Djan (2010) (Ohene-Djan, 2010), Hetzroni and Tannous (2004) (Hetzroni and Tannous, 2004), Hirano et al. (2010) (Hirano et al., 2010), De Leo and Leroy (2008) (De Leo and Leroy, 2008) and Cihak et al. (2012) (Cihak et al., 2012) proposed methodologies to develop communication in a social context, by providing tools to socialize by showing social situations or means to send messages, or to encourage spontaneous vocalizations. And finally, Rodrìguez et al (2004) (Gea-Megías et al., 2004) used an approach to teach the children communicating by showing them casual situations, focusing on the development of the cognitive component as a way of increasing vocabulary and social imagination skills.

In summary, we address research studies and tools according to the following taxonomy:

1. Number of Vocalizations: Studies targeted at motivating the number of vocalizations produced by the children, which is of upmost importance in children that can't speak.

2. Vocabulary Expansion: Authors that try to increase the number of words that the children recognize and use. Communication in children with ASD can be affected by their cognitive abilities, which is a severe impairment that is usually found in children with ASD.

3. Communication in a Social Context: Work that tries to deliver some examples to children on how to socialize, or engage them in some computermediated situations, in order for the children to learn how to react and feel more comfortable when confronted in real life.

4. General Communication Learning: Authors that try to develop cognitive abilities in order to improve several communication impairments. This can help the children to learn more vocabulary, or to prepare them to face different situations.

5. Commercial Tools: Tools that are available for purchase or free download for children with ASD or other types of impairments, without having any research associated, and having only the purpose to answers the users' needs.

\subsection{Number of Vocalizations}

Several studies are focused on developing the children vocalization abilities, but each author has followed a different approach. A simpler approach came with the work by Trying a more social way to motivate vocalizations, Simpson et al. (2004) (Simpson et al., 2004) studied a way to create spontaneous verbal greetings to peers with examples and counter examples of the social skill: sharing, following tutor directions, and social greetings. The approach combined video and computer based instructions using HyperStudio 3.2. The software had several screens that provided written instructions, synthetized speech and video examples. The child was able to select which content to watch and had to discriminate the examples from the counter examples displayed in the videos. Results showed an increase in targeted social skills, and this study has an interesting approach since it tries to develop social skills indirectly, through the communication development. But it's still a limited approach, since it doesn't give the possibility for the children to get to know somehow other peers, and directly exchange messages with them as a safe method to communicate for them. It has a gap between learning how to communicate, and how to physically socialize with peers.

As a more comprehensive approach, which tried to create a package of tools, was proposed by Hailpern et al. (2009) (Hailpern et al., 2009) by presenting the Spoken Impact Project Software (SIPS). In their approach, the authors tried to understand how the effect of audio and visual feedback can encourage vocalizations of children with ASD, with aspects present in previous approaches and with positive results. The SIPS generates audio and visual feedback directly related with the amount of external noise produced by the children and detected by the system, for example, a square shape showed in the screen might change to a round shape according to a sound it captured. The results give us strong indications that audio and visual feedback does encourage spontaneous speech-like vocalizations in low-functioning children with ASD. While using the program, they found that children also have distinct and specific preferences for feedback, stating that in the future they might need individual customization.

\subsection{Vocabulary Expansion}

Another line of research has been concerned with the increase of the vocabulary possessed by children, and although some aspects are somehow similar to those found in the previously described studies, there are different views on how to motivate children, by using other types of stimuli.

A similar study focused on stimulating children with visual and auditory stimuli was developed by Moore and Calvert (2000) (Moore and Calvert, 2000), which analyzed the impact of computers on the extension of the vocabulary of the child, by developing a software program that builds upon behavioral 
learning principles to enhance the vocabulary skills of children with autism. The software provides animations and interesting sounds as a reinforcement when correct responses to the given commands are provided by the child. The results are positive as in the previous study, showing that children pay more attention when using the computer than with the tutor, and that they felt more motivated to continue the study using the computer. An important conclusion is that the number of correct words identified increased. This study doesn't disclose many details about the software, making it somehow suggestive and difficult to evaluate in terms of the adopted methodology.

Still focusing on vocabulary development, but using a different approach, Bosseler and Massaro (2003) (Bosseler and Massaro, 2003) used a software called Baldi which is a $3 \mathrm{D}$ language tutor, qualified to help in learning speech and reading through the association between pictures and spoken words. According to the authors, Baldi has been successful in teaching vocabulary and grammar to children with ASD and to children with hearing problems also they tried to understand if using the tutors face helped increasing the success in the learning process, so they performed experimental evaluation with and without the tutors face. They asked children to take pictures of objects and surroundings at home, which were then incorpored in the lessons. In the results, the number of vocabulary words increased, and the authors concluded that the positive results were due to the lessons with Baldi. Using an embodied agent might be a positive reinforcement since can more easily relate with a non-human character. But with time, based on Putnam's findings (Putnam and Chong, 2008), children may get bored with the agent itself, leading to loss of interest. This could mean that over time the lessons may be increasingly strained, which ultimately leads to decreased motivation and possibly to the reduction of visible results.

Later, Massaro and Bosseler (2006) (Massaro and Bosseler, 2006) also used Baldi as software to conduct tests to teach vocabulary and grammar by identifying pictures. Here the program provided exercises in every lesson with a unique set of items appropriate for the children's vocabulary, knowledge, and abilities. In each lesson they could use the Baldi agent or only the spoken word. During the exercises the child had to choose the correct image given the word that was said, or to choose certain areas of the image according to what was asked. In some exercises they had positive reinforcements with a happy face, or a sad face for incorrect answers. At some point, the child was asked to vocalize the name of the item or its function. Results showed that there were more correct answers with Baldi, than with the audio alone and in the overall the correct answers increased. Although this approach using Baldi it is different from the Bosseler and Massaro (2003) (Bosseler and Massaro, 2003) study, the two show that is possible to test several approaches with the same tool, but the problems listed above of the possible decrease in motivation still apply here, and the results are clear in stating that using the agent is more beneficial than without it.

A suggestive test using rich slides created in Power Point was developed by Coleman-Martin et al. (2005) (Coleman-Martin et al., 2005). In their work, the authors focused on developing the capacity to identify vocabulary words, by starting to show a first slide with the target word written and with the corresponding audio vocalization. Then the subsequent slides presented words by phonemes, for the child to repeat, which was then followed by a positive reinforcement picture, and a sonorous "Excellent". The last slide presented the whole word again. The results showed an increase in vocabulary words, but they tested under three conditions: a) tutor only, (b) tutor plus computer-aided protocol, and (c) computer-aided protocol only. Although using Power Point gives the possibility to easily change all contents and structure, regularly creating and updating slideshows fin-tuned to the childrens needs can involve a considerably large and time consuming effort. Another possibility is that they always use the same document, but that raises the question previously highlighted in another study about the potential loss of interest due to habituation by the child.

Finally, Hetzroni and Shalem (2005) (Hetzroni and Shalem, 2005) tried a more simple approach focused on the vocabulary development, after a study targeted at the development of communication skills in social context (we will detail this study in the next section). The authors developed a tool to implement a 7-step gradual fading protocol, where at first it shows the picture of a food item with the name on it, and then the pictures fade out until only the name is visible. They also used emoticons as a reinforcement. Later, in the classroom setting, children were asked to point out the food items that were written. Results show that correct matches between the text and the food items improved for all participants, but the software shows limited resources and lacks any kind of engaging contents for children. 


\subsection{Communication in a Social Context}

With a more social approach, Hetzroni and Tannous (2004) (Hetzroni and Tannous, 2004) investigated the possibility of enhancing communication functions of children with autism, using software developed by them. They focused on delayed echolalia, immediate echolalia, irrelevant speech, relevant speech, and communicative initiations. The software showed animations of daily routines, and in each one an animation of an adult asking a question related with the current routine. After the child selects the response from a given list, an animation is shown with the results of the selected answer. The results were positive, evidencing a decrease in delayed echolalia, in immediate echolalia, and in irrelevant speech, together with an increase in relevant speech communication initiations. Also, this study showed that children were able to transfer the knowledge from the experimental settings to their real world routines. Over time, children train all possible situations, repeating already trained scenarios, which may lead to a decline in their interest and communication skills.

Woodcock and Woolner (2007) (Woodcock and Woolner, 2007) describe a low cost and user-tuned polysensory environment developed for children with ASD; their work, called inserted in Project Spectrum, which tries to engage children with ASD in using digital technologies. This project has several modules that try to address the whole triad of impairments, by providing a set of predefined interactive activities that are considered to be engaging for children with ASD. It has the concern of including fun and engaging content, leading children to actively seek the use of the software. Using interactive activities, the authors want to provide children with opportunities to communicate and encourage spontaneous vocalizations. One of the drawbacks of this project is the fact that it requires a demanding experimental setup: video cameras for motion tracking, a microphone, lights, a projector, speakers, and a computer. This setup can be very expensive, it requires tutors to learn an extensive set of skills to use it, and it doesn't provides implified modules for message exchange between users.

Devised as a more practical approach, De Leo and Leroy (2008) (De Leo and Leroy, 2008) use a smartphone as a mean to enhancing communication and social skills. They developed a tool called PixTalk ${ }^{1}$, which gives the possibility of choosing from any image placed in the program (that can be customized according to the user' interests), write

\footnotetext{
${ }^{1}$ http://www.communicationautism.com
}

a message, and send it to another user. This is a conceptually approach when compared to ours, since they try to meet the interests and preferences of the users and let them communicate in the way in which they feel more comfortable with. This project evolved to a commercial tool called I Click I Talk 2 and currently has more features than the one used in the study. We were not able to have access to the results of this study, but since a similar approach was followed, we thought that was important to make a reference. Despite the message functionality being similar, it still lacks all the other functionalities and ease of content access and customization, like videos or images to help motivate tool usage and sharing preferences.

Thinking more specifically in a classroom environment and its requirements, often forgotten in these studies, Hirano et al. (2010) (Hirano et al., 2010) developed vSked, an interactive and collaborative visual scheduling system, designed for elementary school classrooms. They define a visual schedule as a set of pictures or words that cues someone to engage in a sequence of activities. They tested it as a timetable with all the children's activities during the day, and each entry on the timetable had an animation associated with that activity. Each student received a notification in their personal devices indicating a location of that activity, or a set of choices for the child to choose the right location. It also provided help when the child chose the wrong answer, or just didn't answer. When the answers were correct, children were rewarded with animations of their liking. The acceptance in the classroom was good both by the children and staff, which reported reductions in the effort required to use visual supports. The results were also positive, showing that the children have interest in using the system, and reached an increase in communication and social interactions in the classroom. But despite proving to be a useful tool, it has a different purpose, and doesn't enable the children to communicate directly with their peers, or visualize their favorite content.

Ohene-Djan (2010) (Ohene-Djan, 2010) developed Winkball, a new Internet-based video messaging and broadcasting technology, designed to support the teaching of oral and visual communication skills in schools. Despite not targetting children with ASD, the author believes that the technology can also be beneficial for this population, since the use of media is usually perceived as interesting and motivating. The author doesn't show any tests or results related with the tool, and therefore it

\footnotetext{
${ }^{2} \mathrm{http}: / / \mathrm{www} . i c l i c k i t a l k . c o m$
} 
is difficult to draw conclusions about its effectiveness.

Millen et al. (2011) (Millen et al., 2011) tried to find ways of supporting communication and collaboration skills for children with autism in the scope of the COSPATIAL project (Communication and social participation: collaborative technologies for interaction and learning) ${ }^{3}$. This project developed a suite of applications composed by two Collaborative Virtual Environments (CVE) to support the enhancement of social skills in children with autism, and two educational technologies in the form of Shared Active Surfaces (SAS). This suite is composed by Block Party CVE (a collaborative puzzle-style game that requires users to communicate and collaborate in order to build a specific tower of blocks in a given pattern), TalkAbout CVE (an avatar with which children must train a social conversation), Join-In Suite SAS (a set of short problematic stories presented to children, that have different solutions either given by the program or suggested by the children, which the child can experience later in a game), and No-Problem! (an activity in which children can explore stages of a social conversation, explore alternatives solutions or suggest new ones). To date, only descriptive information about the project and its goals is available, meaning that there are no results available. Still, we considered important to make a reference to such a project.

Finally, Cihak et al. (2012) (Cihak et al., 2012) tried a simple approach consisting on the evaluation of PECS used in conjunction with Video Modeling (VM) as a technique to increase independent communicative interactions in children with limited to no verbal communication skills. In this study, the authors use video to teach what would normally be taught with PECS. Tests show that as a result of using VM in conjunction with PECS, all students increased independent communication interactions, and the students rate of learning was quicker when using VM. Still, this technique is very limited in giving the children a comprehensive set of tools to enable learning of communication skills, and communicating with others.

\subsection{General Communication Learning}

Rodrìguez et al (2004) (Gea-Megías et al., 2004) propose the Sc@ut Communicator, an Augmentative and Alternative Communication (AAC) designed to help people with communication problems, such as subjects with ASD dysphasia and brain paralysis. The communicator runs in several portable de-

\footnotetext{
${ }^{3} \mathrm{http}: / /$ cospatial.fbk.eu
}

vices (pocket PCs, mobile phones, video game consoles and computers), and uses hypermedia templates with sounds and pictures to improve learning. Sc@ut shows a structure of templates with images, similar to PECS. Images can be pictograms, photos or drawings, which represent objects that the user can ask for, or actions that can be carried out. When an image is selected, an associated sound previously stored is played. This sound, which can be a word or a sentence, represents a reinforcement for the user and allows tutors to know what the user wants. It also provides links between templates, when a specific image is selected in it. This interaction allows the construction of structured sentences, or the classification of actions and elements. They also have a separate tool called Sc@ut Generator (Gea-Megías et al., 2004), which allows the Communicator to be customized "ad-hoc", while the child is progressing in his learning activities, thus adapting dynamically to the progress of the child, unlike other more rigid AAC systems. This way tutors can change content and interaction, adapting them to specific educational contexts, so that the software can be used as a support in learning process. This tool tries to adapt to the children' needs and give them a way to easily learn context-specific situations, but it doesn't allow them communicate directly with other people.

\subsection{Commercial Tools}

There are a few applications in the market that despite not being the matter of a study for which we can assess experimental results, are still important to look and evaluate. Some of them have been specifically developed for people with ASD, trying to help with the cognitive and communication impariments. Other tools are broad spectrum and aimed at populations with very distinct needs, although they are also applicable to people with ASD. As in the previous section we are going to analyze and describe these tools.

\section{Zac Browser}

The Zone for Autistic Children Browser (Zac Browser $)^{4}$, is a multimedia software developed for children with ASD. This tool was inspired in an autistic child named Zachary, which was unable to use his computer using regular computer software tools (e.g. Internet Explorer, Windows Messenger, etc.). Faced with these difficulties, his grandfather decided to develop the Zac Browser as a software completely adapted to Zachary's needs with a more visual navigation, and safer to use due to parental

\footnotetext{
${ }^{4}$ http://zacbrowser.com
} 
control. This software is especially designed for recreational and entertainment use, and as such, it does not explore the interpersonal communication specificities. Furthermore, tutors are limited in terms of content adaptation and software configuration, given that they are bound to the pre-defined contents. Furthermore, the tool depends on Internet connection to access most contents, which may not available in every school.

\section{Boardmaker}

Boardmaker ${ }^{5}$ is a suite of applications that enables the adaptation of learning materials to the needs of each child. The program has a large database of symbols they call Picture Communication Symbols $(\mathrm{PCS})^{\mathrm{TM}}$, and allows the users to create any kind of customized material, such as schedules, manual communication boards, books, teaching materials, etc. The material can be used in the computer or printed, making this tool usefull to create materials for teaching, however, this software is designed for the tutor and was not conceived to be used by children.

\section{TeachTown}

TeachTown ${ }^{6}$ is a Computer-Assisted Instructional (CAI) program designed for developmental ages between 2-6 years, specifically to meet needs of ASD population. The program includes a comprehensive curriculum that aligns to standardized measures, which integrated the following learning domains: 1) Language Development; 2) Social and Emotional Skills; 3) Adaptive Skills; 4) Cognitive Skills; 5) Language Arts; and 6) Mathematics. The curriculumdepends on the performance and is adjusted for each student. Tutors can customize the curriculum to meet Individual Educational Plan (IEP) goals. TeachTown also collects data from the children usage and produces reports, to track the student progress. This tool is one of the most complete applications for children with ASD currently available, and extensive research has proven that an ICT tool like TeachTown has beneficial results. However, this tool is not completely customizable, and it is not targetting all the children preferences, which can have poor effects in their attention and engagement. Furthermore, this tool was devised for individual use, and lacks features that can promote communication and social interaction skills.

\section{Tablet Applications}

With the advent of the iPad, several new applica-

\footnotetext{
${ }^{5} \mathrm{http} / / /$ www.mayer-johnson.com/boardmaker-software

${ }^{6} \mathrm{http}: / /$ web.teachtown.com/
}

tions have been created for children to use in tablet devices, motivated by the ease-of-use of the touchscreen. In fact, touchscreens brought new possibilities, since users with cognitive and motor impairments have an easier way to interact with the computer. Instead of using the usual keyboard and mouse, which requires a higher coordination or a greater understanding of its operation that the child sometimes does not have, tablets enable a more natural interaction.

There are some applications that became a reference in the autism area such as Proloquo2 $\mathrm{Go}^{7}$, MyTalk $^{8}$, Grace ${ }^{9}$ and iCommunicate ${ }^{10}$. Despite being different applications, all of them use the PECS format to communicate; the child can choose the picture that best translates what he/she is trying to say, or combine several pictures to write a phrase or story. Some applications also have voice speech that reads what the children write. Most of them are paid, which means that these applications are developed with a commercial purpose in mind, and they only allow a local and physical interaction using the tablet with their peers.

\section{CONCLUSIONS}

In the overall, existing approaches and methodologies proposed to date focus on self-development and don't show much concern towards enabling children to communicate with each other, or adapting the tool to the user, or deliver any kind of courseware. Most of the work found to data is inspired in PECS, which is a simple system that children understand, and is simple to use, and even in the cases where user-tuned content customization is possible, the procedures to do so require the tutor to acquire specific knowledge and the customization options are limited. After a closer look at the studies focused on communication, we can understand the different concerns and tactics used. In vocalizations studies we see that audio is the most important feature to be used, as these approaches try to resort to imitation as a way to increase the user confidence in using speech. We can see that only two use images, and only one uses video, from which we can infer that using this kind of media to motivate speech imitation might be intentionally avoided, in order to prevent any stress caused by the present or interaction with people (even if it is through the

\footnotetext{
${ }^{7} \mathrm{http} / / / \mathrm{www}$. assistiveware.com/product/proloquo2go

${ }^{8} \mathrm{http}: / /$ www.mytalktools.com

${ }^{9}$ http://www.graceapp.com

${ }^{10} \mathrm{http}: / /$ www.grembe.com/icommunicate
} 
computer). In general at the end of the tasks, all experimental setups present animations to the user as a positive feedback reward for their efforts, motivating them. As previously described, in this type of tests, direct human interactions are not used, and so there are no social components involved. Furthermore, except for one of the studies, none of the proposed approaches gives the possibility to change any aspect of the tool; only Simpson et al. (2004) (Simpson et al., 2004) enables content to be changed. It is also important to highlight that most of the platforms used in the presented studies are not developed specifically for the study; most of the features addressed by our work are not be present because the researchers simply were not able to experiment with them or just haven't felt the need to use it. In the vocabulary expansion field, where most studies are found, most of the conclusions stated for vocalizations also apply. We see that social communication continues to be missing, also probably to avoid stressing the children. Media support is found to be very important; most of the studies support mainly images, audio and animations, although videos still don't seem to have much importance, since most of the studies don't support or use them in the tests. The main difference is that most of the tools enable the possibility to change content (Heimann et al., 1995; Moore and Calvert, 2000; Bosseler and Massaro, 2003; Coleman-Martin et al., 2005; Hetzroni and Shalem, 2005), which might show that researchers realized the importance of adapting the tool to the user, in order to achieve better outcomes. These results also apply to the studies that focus on developing communication in a social context, and where the only obvious difference is the inclusion of message exchange in three of the studies (De Leo and Leroy, 2008; Ohene-Djan, 2010; Millen et al., 2011). Other commercial tools include more functionalities supporting most media formats, messages and the possibility to change a few aspects of the content and some functionalities of the tool itself (a feature not found in any of the other studies). However, these features are motivated by the need to address the requirements of a broader spectrum of target user groups (e.g. people with motor impairments), which in most cases does not account for aspects that are core to the ASD population, such as having engaging and fun contents and interfaces. But they don't show much concern about being able to put the children to communicate with each other, or adapting the tool to the user, or deliver any kind of courseware. All of the studies explore the use of multimedia, which shows us that this is the most effective way of capturing the attention of the children and help in the development of new communication skills. In general, we see that two of the main lines of research, the enhancement of the children's vocalizations and vocabulary, are using a more mechanic approach supported by imitation combined with a visual and audio positive reinforcement. This kind of approach, despite helping children to talk, doesn't help them to communicate with other people or offers ways to do it in a comfortable way for them. These tools also have a high probability of leading to lack of interest over time, since the contents and interface are always the same, not giving the possibility for tutors to change anything in order to stimulate a more constant motivation. This same reason makes the tool less fun for the children, which is an important factor that is pointed out in Putnam's study (Putnam and Chong, 2008) as one of the main causes of abandonment in computer-based interventions. These issues arise due to the fact that little power is given to the tutors and tutors, to change either contents, or the tool interface, easily becoming outdated. None of the studies focuses on the tutors' or parents' deep knowledge of their child's interests and needs, which should be one of the best ways to get through to the child and prepare usertuned contents in a way that is more engaging and positive for their personal development. New possibilities have emerged with touchscreen technologies (e.g. smartphones and tablets), bringing new opportunities to users (usually paid), and mostly designed to help parents in the interaction with their children. Typically, most applications arising nowadays show concerns about enabling the children to compose stories or phrases to communicate, using custom keyboards or touchscreen, to facilitate the handling of the and to enhance self-communication through and message composition.

\section{ACKNOWLEDGEMENTS}

This work has been partially funded by Fundação Portugal Telecom, ASUS Portugal, and by the Fundação para a Ciência e Tecnologia (FCT) under the grant SFRH/BD/65248/2009 and INESC-ID multiannual funding under project PEst-OE/EEI/LA0021/2013.

\section{REFERENCES}

Bernard-Opitz, V., Sriram, N., and Sapuan, S. (1999). Enhancing vocal imitation in children with Autism using the IBM Speech Viewer. Autism, 3(2):131-147.

Bondy, A. and Frost, L. (2001). The Picture Exchange Communication System. Behavior Modification, 25(5):725-744. 
Bosseler, A. and Massaro, D. W. (2003). Development and evaluation of a computer-animated tutor for vocabulary and language learning in children with autism. Journal of Autism an Developmental Disorders, 33(6):653-672.

Cihak, D. F., Smith, C. C., Cornett, A., and Coleman, M. B. (2012). The use of video modeling with the Picture Exchange Communication System to increase independent communicative initiations in preschoolers with Autism and developmental delays. Focus on Autism and Other Developmental Disabilities, 27(1):3-11.

Coleman-Martin, M. B., Heller, K. W., Cihak, D. F., and Irvine, K. L. (2005). Using computer-assisted instruction and the nonverbal reading approach to teach word identication. Focus on Autism and Other Developmental Disabilities, 20(2):80-90.

De Leo, G. and Leroy, G. (2008). Smartphones to facilitate communication and improve social skills of children with severe autism spectrum disorder: special education teachers as proxies. In Proceedings of the 7th International Conference on Interaction Design and Children (IDC), pages 45-48, New York, NY, USA. ACM.

Francis, P., Balbo, S., and Firth, L. (2009). Towards codesign with users who have autism spectrum disorders. volume 8, pages 123-135. Springer Berlin / Heidelberg.

Gea-Megías, M., Medina-Medina, N., RodríguezAlmendros, M., and Rodríguez-Fórtiz, M. (2004). Sc@ut: Platform for communication in ubiquitous and adaptive environments applied for children with Autism. In Stary, C. and Stephanidis, C., editors, User-Centered Interaction Paradigms for Universal Access in the Information Society, volume 3196 of Lecture Notes in Computer Science, pages 50-67. Springer Berlin Heidelberg.

Hailpern, J., Karahalios, K., and Halle, J. (2009). Creating a spoken impact: Encouraging vocalization through audio visual feedback in children with ASD. In Proceedings of the 27th International Conference on Human Factors in Computing Systems (CHI), pages 453-462, New York, NY, USA. ACM.

Heimann, M., Nelson, K. E., Tjus, T., and Gillberg, C. (1995). Increasing reading and communication skills in children with Autism through an interactive multimedia computer program. Journal of Autism and Developmental Disorders, 25(5):459-80.

Hetzroni, O. and Tannous, J. (2004). Effects of a computerbased intervention program on the communicative functions of children with Autism. Journal of Autism and Developmental Disorders, 34(2):95-113.

Hetzroni, O. E. and Shalem, U. (2005). From logos to orthographic symbols: A multilevel fading computer program for teaching nonverbal children with Autism. Focus on Autism and Other Developmental Disabilities, 20(4):201-212.

Hirano, S. H., Yeganyan, M. T., Marcu, G., Nguyen, D. H., Boyd, L. A., and Hayes, G. R. (2010). vSked: evaluation of a system to support classroom activities for children with autism. In Proceedings of the 28th International Conference on Human Factors in Computing Systems (CHI), pages 1633-1642, New York, NY, USA. ACM.

Massaro, D. W. and Bosseler, A. (2006). Read my lips: The importance of the face in a computer-animated tutor for vocabulary learning by children with autism. Autism, 10(5):495-510.

Menzies, R. (2011). Developing for autism with usercentred design. In Proceedings of the 13th ACM International Conference on Computers and Accessibility (ASSETS), pages 313-314, New York, NY, USA. ACM.

Millen, L., Hawkins, T., Cobb, S., Zancanaro, M., Glover, T., Weiss, P. L., and Gal, E. (2011). Collaborative technologies for children with autism. In Proceedings of the 10th International Conference on Interaction Design and Children (IDC), pages 246-249, New York, NY, USA. ACM.

Moore, M. and Calvert, S. (2000). Vocabulary acquisition for children with Autism: Teacher or computer instruction. Journal of Autism and Developmental Disorders, 30(4):359-362.

Morris, R. R., Kirschbaum, C. R., and Picard, R. W. (2010). Broadening accessibility through special interests: a new approach for software customization. In Proceedings of the 12th ACM International Conference on Computers and Accessibility (ASSETS), pages 171178, New York, NY, USA. ACM.

Nelson, K. E. and Prinz, P. M. (1991). Alpha interactive language series/gator super sentences (computer software). Warriors Mark, PA: Super Impact Images.

Ohene-Djan, J. (2010). Winkball for schools: An advanced video modelling technology for learning visual and oral communication skills. In 10th International Confernece on Advanced Learning Technologies (ICALT), pages 687-689. IEEE.

Parsons, C. L. and La Sorte, D. (1993). The effect of computers with synthesized speech and no speech on the spontaneous communication of children with autism. Australian Journal of Human Communication Disorder, 21:12-31.

Putnam, C. and Chong, L. (2008). Software and technologies designed for people with autism: what do users want? In Proceedings of the 10th ACM International Conference on Computers and Accessibility (ASSETS), pages 3-10, New York, NY, USA. ACM.

Simpson, A., Langone, J., and Ayres, K. M. (2004). Embedded video and computer based instruction to improve social skills for students with Autism. Education and Training in Developmental Disabilities, 39(3):240 252.

Woodcock, A. and Woolner, A. (2007). Facilitating communication, teaching and learning in children with an ASD: Project spectrum. In Proceedings of the IEEE on Development and Learning (ICDL), pages 59-63. IEEE. 